International Journal of Pure and Applied Mathematics

Volume 93 No. 4 2014, 511-523

ISSN: 1311-8080 (printed version); ISSN: 1314-3395 (on-line version)

url: http://www.ijpam.eu

doi: http://dx.doi.org/10.12732/ijpam.v93i4.3

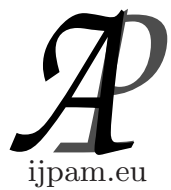

\title{
VAGUE SOFT MULTISET THEORY
}

\author{
Khaleed Alhazaymeh ${ }^{1}$, Nasruddin Hassan ${ }^{2} \S$ \\ ${ }^{1,2}$ School of Mathematical Sciences \\ Universiti Kebangsaan Malaysia \\ 43600 UKM, Bangi Selangor, MALAYSIA
}

\begin{abstract}
In this paper we introduce the concept of vague soft multiset which is an extension of soft set. We define the notion of equality, subset hood and nullity vague soft multisets. We illustrate several operations with examples and an application of vague soft multiset theory to a decision making problem.
\end{abstract}

AMS Subject Classification: 03B52, 03E72

Key Words: soft multiset theory, vague set, vague soft set

\section{Introduction}

Decision making problems are usually solved by goal programming [1], [2], [3], [4], [5], [6], [7], [8], [9], [10], [11], [12] or data envelopment analysis [13], [14], [15]. Fuzzy set [16] was introduced as a mathematical tool to solve the problem and vagueness in everyday life. This was extended to fuzzy soft set [17], [18], [19], [20], [21] and vague soft sets [22], [23], [24], [25], [26], [27], [28], [29], [30] and used to solve genetic algorithms [31], [32] and further extended to multi Q-fuzzy [33]. In this paper, we introduce the concept of vague soft multiset and some operations with illustrative example on decision making.

Received: November 26, 2013

(C) 2014 Academic Publications, Ltd.

${ }^{\S}$ Correspondence author url: www.acadpubl.eu 


\section{Preliminaries}

Molodtsov [34] defined soft set in the following way. Let $U$ be a universal set and $E$ be a set of parameters. Let $P(U)$ denotes the power set of $U$ and $A \subseteq E$.

Definition 2.1. (see [34]) A pair $(F, A)$ is called a soft set over $U$, where $F$ is a mapping $F: A \rightarrow P(U)$.

In other words, a soft set over $U$ is a parameterized family of subsets of the universe $U$. For $\varepsilon \in A, F(\varepsilon)$ may be considered as the set of $\varepsilon$-approximate elements of the soft set $(F, A)$.

Definition 2.2. (see [17]) Let $\left\{U_{i}: i \in I\right\}$ be a collection of universes such that $\bigcap_{i \in I} U_{i}=\phi$ and let $\left\{E_{U_{i}}: i \in I\right\}$ be a collection of sets of parameters. Let $U=\prod_{i \in I} P\left(U_{i}\right)$ where $P\left(U_{i}\right)$ denotes the power set of $U_{i}, E=\prod_{i \in I} E_{U_{i}}$ and $A \subseteq E$. A pair $(F, A)$ is called a soft multiset over $U$, where $F$ is a mapping given by $F: A \longrightarrow U$

In other words, a soft multiset over $U$ is a parameterized family of subsets of $U$. For $\varepsilon \in A, F(\varepsilon)$ may be considered as the set of $\varepsilon$-approximate elements of the soft multiset $(F, A)$. Based on the above definition, any change in the order of universes will produce a different soft multiset.

Definition 2.3. (see [17]) For any soft multiset $(F, A)$, a pair $\left(e_{U_{i} j}, F_{e_{U_{i}, j}}\right)$

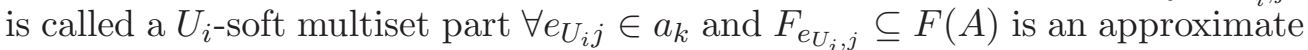
value set, where $a_{k} \in A, k=\{1,2, \ldots, n\}, i=\{1,2, \ldots, m\}$ and $j=\{1,2, \ldots, r\}$.

Definition 2.4. (see [17]) For two soft multisets $(F, A)$ and $(G, B)$ over $U,(F, A)$ is called a soft multisubset of $(G, B)$ if:

(i) $A \subseteq B$ and,

(ii) $\forall e_{U_{i} j} \in a_{k},\left(e_{U_{i} j}, F_{e_{U_{i}, j}}\right) \subseteq\left(e_{U_{i} j}, G_{e_{U_{i}, j}}\right)$,

where $a_{k} \in A, k=\{1,2, \ldots, n\}, i=\{1,2, \ldots, m\}$ and $j=\{1,2, \ldots, r\}$.

This relationship is denoted by $(F, A) \tilde{\subseteq}(G, B)$.

\section{Vague Soft Multiset}

In this section, we introduce the definition of a vague soft multiset, and its basic operations such as complement, union and intersection with examples.

Definition 3.1. Let $\left\{U_{i}: i \in I\right\}$ be a collection of universes such that $\bigcap_{i \in I} U_{i}=\phi$ and let $\left\{E_{U_{i}}: i \in I\right\}$ be a collection of sets of parameters. Let $U=$ $\prod_{i \in I} V\left(U_{i}\right)$ where $V\left(U_{i}\right)$ denotes the set of all vague subset of $U_{i}, E=\prod_{i \in I} E_{U_{i}}$ 
and $A \subseteq E$. A pair $(F, A)$ is called a vague soft multiset over $U$, where $F$ is a mapping given by $F: A \rightarrow U$.

Definition 3.2. For any vague soft $\operatorname{multiset}(F, A)$, a pair $\left(e_{U_{i} j}, F_{e_{U_{i}, j}}\right)$ is called a $U_{i}$-vague soft multiset part $\forall e_{U_{i} j} \in a_{k}$ and $F_{e_{U_{i}, j}} \subseteq F(A)$ is a vague approximate value set, where $a_{k} \in A, k=\{1,2, \ldots, n\}, i=\{1,2, \ldots, m\}$ and $j=\{1,2, \ldots, r\}$.

Definition 3.3. For two vague soft multisets $(\tilde{F}, A)$ and $(\tilde{G}, B)$ over $U$, $(\tilde{F}, A)$ is called a vague soft multisubset of $(\tilde{G}, B)$ if:

(i) $A \subseteq B$ and,

(ii) $\forall e_{U_{i} j} \in a_{k},\left(e_{U_{i} j}, F_{e_{U_{i}, j}}\right)$ is a vague subset of $\left(e_{U_{i} j}, G_{e_{U_{i}, j}}\right)$, where $a_{k} \in A, k=\{1,2, \ldots, n\}, i=\{1,2, \ldots, m\}$ and $j=\{1,2, \ldots, r\}$.

This relationship is denoted by $(F, A) \tilde{\tilde{C}}(G, B)$. In this case $(G, B)$ is called a soft multisuperset of $(F, A)$.

Definition 3.4. The complement of a vague soft multiset $(F, A)$ is denoted by $(F, A)^{c}$ and is defined by $(F, A)^{c}=\left(F^{c}, A\right)$, where $F^{c}: A \longrightarrow U$ is a mapping given by $F^{C}(\alpha)=U-F(\neg \alpha), \forall \alpha \in \neg A$.

\section{An Application in Decision Making}

In this section we recall the algorithm designed for solving a fuzzy soft multiset theoretic approach to decision making problem presented by Alkhazaleh et al. [16]. We note here that we will use the abbreviation (RMA) for Roy and Maji's Algorithm.

(a) Input the vague soft multiset $(H, C)$ which is introduced by making any operations between $(F, A)$ and $(G, B)$.

(b) Apply RMA to the first vague soft multiset part in $(H, C)$ to get $S_{k_{1}}$.

(c) Redefine the vague soft multiset $(H, C)$ by keeping all values in each row where $S_{k_{1}}$ is maximum and replacing the values in the other rows by zero.

(d) Apply RMA to the second vague soft multiset part in $(H, C)_{1}$ to get $S_{k_{2}}$.

(e) Redefine the vague soft multiset $(F, A)_{1}$ by keeping the first and second parts and apply the method in step 3 to the third part. 
(f) Apply RMA to the third vague soft multiset part in $(H, C)_{2}$ to get $S_{k_{3}}$.

(g) The decision is $\left(S_{k_{1}}, S_{k_{2}}, S_{k_{3}}\right)$.

Let $U_{1}=\left\{h_{1}, h_{2}, h_{3}\right\}, U_{2}=\left\{c_{1}, c_{2}, c_{3}\right\}$ and $U_{3}=\left\{v_{1}, v_{2}, v_{3}\right\}$ be the sets of "venue", "machine" and "trucks", respectively. Let $\left\{E_{U_{1}}, E_{U_{2}}, E_{U_{3}}\right\}$ be a collection of sets of decision parameters related to the above universes, where:

$E_{U_{1}}=\left\{e_{U_{1}, 1}=\right.$ in Kuala Lumpur, $e_{U_{1}, 2}=$ Bangi, $e_{U_{1}, 3}=$ Shah Alam $\}$.

$E_{U_{2}}=\left\{e_{U_{1}, 1}=\right.$ synchronous machines, $e_{U_{1}, 2}=$ DC machines, $e_{U_{1}, 3}=$ induction or asynchronous machines $\}$.

$E_{U_{3}}=\left\{e_{U_{1}, 1}=\right.$ light trucks, $e_{U_{1}, 2}=$ medium trucks, $e_{U_{1}, 3}=$ heavy trucks $\}$.

$A=\left\{a_{1}=\left(e_{U_{1}, 1}, e_{U_{2}, 1}, e_{U_{3}, 1}\right)\right.$,

$a_{2}=\left(e_{U_{1}, 1}, e_{U_{2}, 3}, e_{U_{3}, 1}\right)$,

$a_{3}=\left(e_{U_{2}}, 1, e_{U_{1}, 2}, e_{U_{3}, 3}\right)$,

$\left.a_{4}=\left(e_{U_{3}}, 1, e_{U_{1}}, 3, e_{U_{3}, 2}\right)\right\}$, and

$B=\left\{b_{1}=\left(e_{U_{1}, 1}, e_{U_{2}, 1}, e_{U_{3}, 1}\right)\right.$,

$b_{2}=\left(e_{U_{1}, 2}, e_{U_{2}}, 1, e_{U_{3}, 1}\right)$,

$b_{3}=\left(e_{U_{1}}, 3, e_{U_{3}, 1}, e_{U_{3}, 2}\right)$,

$\left.b_{4}=\left(e_{U_{3}, 1}, e_{U_{1}, 3}, e_{U_{3}, 2}\right)\right\}$.

Suppose that a person wants to buy objects from the sets of given objects with respect to the sets of choice parameters. Let there be two observations $(F, A)$ and $(G, B)$ by two experts $Y_{1}$ and $Y_{2}$ respectively. Let

$$
\begin{gathered}
(F, A)=\left\{a_{1},\left\{\frac{h_{1}}{[0.5,0.5]}, \frac{h_{2}}{[0.6,0.7]}, \frac{h_{3}}{[0.8,0.9]}, \frac{h_{4}}{[1,1]}\right\},\right. \\
\left.\left\{\frac{c_{1}}{[0.6,0.8]} \frac{c_{2}}{[0.5,0.5]} \frac{c_{3}}{[0.6,0.9]}\right\},\left\{\frac{v_{1}}{[0.8,0.9]} \frac{v_{2}}{[0.7,0.8]} \frac{v_{3}}{[0.2,0.6]}\right\}\right), \\
a_{2},\left\{\frac{h_{1}}{[0.2,0.7]}, \frac{h_{2}}{[0.1,0.1]}, \frac{h_{3}}{[0,0]}, \frac{h_{4}}{[0.9,0.9]}\right\},\left\{\frac{c_{1}}{[0.5,0.5]} \frac{c_{2}}{[0.7,0.7]} \frac{c_{3}}{[0.2,0.3]}\right\}, \\
\left.\left\{\frac{v_{1}}{[0.1,0.8]} \frac{v_{2}}{[0.5,0.9]} \frac{v_{3}}{[0.2,0.8]}\right\}\right), a_{3},\left\{\frac{h_{1}}{[0.3,0.6]}, \frac{h_{2}}{[0.7,0.8]}, \frac{h_{3}}{[0.9,0.9]}, \frac{h_{4}}{[0.1,0.9]}\right\}, \\
a_{4},\left\{\frac{v_{1}}{[0.4,0.7]}, \frac{v_{2}}{[0.8,0.9]}, \frac{v_{3}}{[0.9,0.9]}, \frac{v_{2}}{[0.7,0.8]} \frac{c_{3}}{[0.6,0.7]} \frac{c_{3}}{[0.3,0.5]}\right\},\left\{\frac{h_{4}}{[0.3,0.6]}\right\},\left\{\frac{c_{1}}{[0,0]} \frac{c_{2}}{[0.8,0.9]} \frac{c_{3}}{[0.6,0.6]} \frac{c_{1}}{[0.5,0.6]}\right\}, \\
\left.\left\{\frac{v_{2}}{[0.4,0.4]} \frac{v_{3}}{[1,1]}\right\}\right),
\end{gathered}
$$




$$
\begin{gathered}
(G, B)=\left\{b_{1},\left\{\frac{h_{1}}{[0.6,0.6]}, \frac{h_{2}}{[0.6,0.9]}, \frac{h_{3}}{[0.2,0.2]}, \frac{h_{4}}{[0,1]}\right\},\right. \\
\left.\left\{\frac{c_{1}}{[0.7,0.7]} \frac{c_{2}}{[0.6,0.9]} \frac{c_{3}}{[0.8,0.9]}\right\},\left\{\frac{v_{1}}{[0.9,0.9]} \frac{v_{2}}{[0.6,0.8]} \frac{v_{3}}{[0.4,0.6]}\right\}\right), \\
b_{2},\left\{\frac{h_{1}}{[0.4,0.4]}, \frac{h_{2}}{[0.1,0.9]}, \frac{h_{3}}{[0,1]}, \frac{h_{4}}{[0.1,1]}\right\},\left\{\frac{c_{1}}{[0.6,0.7]} \frac{c_{2}}{[0.8,0.8]} \frac{c_{3}}{[0.2,0.6]}\right\}, \\
\left.\left\{\frac{v_{1}}{[0.2,0.9]} \frac{v_{2}}{[0.5,1]} \frac{v_{3}}{[0.5,0.5]}\right\}\right), b_{3},\left\{\frac{h_{1}}{[0.4,0.5]}, \frac{h_{2}}{[0.8,0.9]}, \frac{h_{3}}{[0.1,0.1]}, \frac{h_{4}}{[0.3,1]}\right\}, \\
\left.\left\{\frac{c_{1}}{[0.2,0.9]} \frac{c_{2}}{[0.7,0.8]} \frac{c_{3}}{[0.4,0.6]}\right\},\left\{\frac{v_{1}}{[0.4,0.7]} \frac{v_{2}}{[0,1]} \frac{v_{3}}{[0,0]}\right\}\right), \\
b_{4},\left\{\frac{h_{1}}{[0.4,0.7]}, \frac{h_{2}}{[0.8,0.9]}, \frac{h_{3}}{[0.9,0.9]}, \frac{h_{4}}{[0.3,1]}\right\}, \\
\left.\left\{\frac{c_{1}}{[0.8,0.9]} \frac{c_{2}}{[0.6,0.6]} \frac{c_{3}}{[0.5,0.6]}\right\},\left\{\frac{v_{1}}{[0.4,0.4]} \frac{v_{2}}{[1,1]} \frac{v_{3}}{[0,0]}\right\}\right) .
\end{gathered}
$$

By using the basic fuzzy union we have

$$
\begin{aligned}
& (F, A) \tilde{\cup}(G, B)=(H, D)=\left\{d_{1},\left\{\frac{h_{1}}{[0.5,0.5]}, \frac{h_{2}}{[0.6,0.7]}, \frac{h_{3}}{[0.2,0.2]}, \frac{h_{4}}{[0,1]}\right\},\right. \\
& \left.\left\{\frac{c_{1}}{[0.6,0.7]} \frac{c_{2}}{[0.5,0.5]} \frac{c_{3}}{[0.6,0.9]}\right\},\left\{\frac{v_{1}}{[0.8,0.9]} \frac{v_{2}}{[0.5,0.5]} \frac{v_{3}}{[0.2,0.6]}\right\}\right) \text {, } \\
& d_{2},\left\{\frac{h_{1}}{[0.4,0.7]}, \frac{h_{2}}{[0.8,0.9]}, \frac{h_{3}}{[0.9,0.9]}, \frac{h_{4}}{[0.3,1]}\right\}, \\
& \left.\left\{\frac{c_{1}}{[0.8,0.9]} \frac{c_{2}}{[0.6,0.6]} \frac{c_{3}}{[0.5,0.6]}\right\},\left\{\frac{v_{1}}{[0.4,0.4]} \frac{v_{2}}{[1,1]} \frac{v_{3}}{[0,0]}\right\}\right), \\
& d_{3},\left\{\frac{h_{1}}{[0.2,0.7]}, \frac{h_{2}}{[0.1,0.1]}, \frac{h_{3}}{[0,0]}, \frac{h_{4}}{[0.9,0.9]}\right\}, \\
& \left.\left\{\frac{c_{1}}{[0.5,0.5]} \frac{c_{2}}{[0.7,0.7]} \frac{c_{3}}{[0.2,0.3]}\right\},\left\{\frac{v_{1}}{[0.1,0.8]} \frac{v_{2}}{[0.5,0.9]} \frac{v_{3}}{[0.2,0.8]}\right\}\right), \\
& d_{4},\left\{\frac{h_{1}}{[0.3,0.6]}, \frac{h_{2}}{[0.7,0.8]}, \frac{h_{3}}{[0.9,0.9]}, \frac{h_{4}}{[0.1,0.9]}\right\}, \\
& \left.\left\{\frac{c_{1}}{[0.7,0.8]} \frac{c_{2}}{[0.6,0.7]} \frac{c_{3}}{[0.3,0.5]}\right\},\left\{\frac{v_{1}}{[0.3,0.6]} \frac{v_{2}}{[0,0]} \frac{v_{3}}{[1,1]}\right\}\right), \\
& d_{5},\left\{\frac{h_{1}}{[0.4,0.4]}, \frac{h_{2}}{[0.1,0.9]}, \frac{h_{3}}{[0,1]}, \frac{h_{4}}{[0.1,1]}\right\}, \\
& \left.\left\{\frac{c_{1}}{[0.6,0.7]} \frac{c_{2}}{[0.8,0.8]} \frac{c_{3}}{[0.2,0.6]}\right\},\left\{\frac{v_{1}}{[0.2,0.9]} \frac{v_{2}}{[0.5,1]} \frac{v_{3}}{[0.5,0.5]}\right\}\right) \text {, } \\
& d_{6},\left\{\frac{h_{1}}{[0.4,0.5]}, \frac{h_{2}}{[0.8,0.9]}, \frac{h_{3}}{[0.1,0.1]}, \frac{h_{4}}{[0.3,1]}\right\},\left\{\frac{c_{1}}{[0.2,0.9]} \frac{c_{2}}{[0.7,0.8]} \frac{c_{3}}{[0.4,0.6]}\right\},
\end{aligned}
$$




$$
\left.\left\{\frac{v_{1}}{[0.4,0.7]} \frac{v_{2}}{[0,1]} \frac{v_{3}}{[0,0]}\right\}\right) .
$$

Apply RMA to the first vague soft multiset part in $(H, D)$ to take the decision from the availability set $U_{1}$ and subtract the truth membership function from the false-membership functions. The tabular representation of the first resultant vague soft multiset part will be as in Table 1 .

Table 1: Representation of $U_{1}$-vague soft multiset part of $(H, D)$

\begin{tabular}{ccccccc}
\hline$U_{1}$ & $d_{1,1}$ & $d_{1,2}$ & $d_{1,3}$ & $d_{1,4}$ & $d_{1,5}$ & $d_{1,6}$ \\
\hline$h_{1}$ & 0 & -0.3 & -0.5 & -0.3 & 0 & -0.1 \\
$h_{2}$ & -0.3 & -0.1 & 0 & -0.1 & -0.8 & -0.1 \\
$h_{3}$ & 0 & 0 & 0 & 0 & -1 & 0 \\
$h_{4}$ & 0 & -0.7 & -0.7 & -0.8 & -0.9 & -0.7 \\
\hline
\end{tabular}

Table 2 is the comparison table for the first resultant vague soft multiset.

Table 2: Comparison table of $U_{1}$-vague soft multiset part of $(H, D)$

\begin{tabular}{ccccc}
\hline$U_{1}$ & $h_{1}$ & $h_{2}$ & $h_{3}$ & $h_{4}$ \\
\hline$h_{1}$ & 6 & 2 & 2 & 6 \\
$h_{2}$ & 4 & 6 & 1 & 5 \\
$h_{3}$ & 5 & 5 & 6 & 5 \\
$h_{4}$ & 1 & 1 & 1 & 6 \\
\hline
\end{tabular}

The row-sum, column-sum, and the score for each $h_{i}$ is shown in Table 3 .

Table 3: Score of $U_{1}$-vague soft multiset part of $(H, D)$

\begin{tabular}{cccc}
\hline$U_{1}$ & Row sum $\left(r_{i}\right)$ & Column sum $\left(t_{i}\right)$ & Membership score $\left(s_{i}\right)$ \\
\hline$h_{1}$ & 16 & 16 & 0 \\
$h_{2}$ & 16 & 14 & 2 \\
$h_{3}$ & 21 & 10 & 11 \\
$h_{4}$ & 9 & 22 & -13 \\
\hline
\end{tabular}


From Table 3, the maximum score is 11 scored by $h_{3}$.

Redefine the vague soft multiset $(H, D)$ by keeping all values in each row where $h_{3}$ is maximum and replacing the values in the other rows by zero.

$$
\begin{gathered}
(H, D)_{1}=\left\{d_{1},\left\{\frac{h_{1}}{[0.5,0.5]}, \frac{h_{2}}{[0.6,0.7]}, \frac{h_{3}}{[0.2,0.2]}, \frac{h_{4}}{[0,1]}\right\},\left\{\frac{c_{1}}{[0,1]} \frac{c_{2}}{[0,1]} \frac{c_{3}}{[0,1]}\right\},\right. \\
\left.\left\{\frac{v_{1}}{[0,1]} \frac{v_{2}}{[0,1]} \frac{v_{3}}{[0,1]}\right\}\right), d_{2},\left\{\frac{h_{1}}{[0.4,0.7]}, \frac{h_{2}}{[0.8,0.9]}, \frac{h_{3}}{[0.9,0.9]}, \frac{h_{4}}{[0.3,1]}\right\}, \\
\left.\left\{\frac{c_{1}}{[0.8,0.9]} \frac{c_{2}}{[0.6,0.6]} \frac{c_{3}}{[0.5,0.6]}\right\},\left\{\frac{v_{1}}{[0.4,0.4]} \frac{v_{2}}{[1,1]} \frac{v_{3}}{[0,0]}\right\}\right), \\
\left.d_{3},\left\{\frac{h_{1}}{[0.2,0.7]}, \frac{h_{2}}{[0.1,0.1]}, \frac{h_{3}}{[0,0]}, \frac{h_{4}}{[0.9,0.9]}\right\},\left\{\frac{c_{1}}{[0,1]} \frac{c_{2}}{[0,1]} \frac{c_{3}}{[0,1]}\right\},\left\{\frac{v_{1}}{[0,1]} \frac{v_{2}}{[0,1]} \frac{v_{3}}{[0,1]}\right\}\right), \\
d_{4},\left\{\frac{h_{1}}{[0.3,0.6]}, \frac{h_{2}}{[0.7,0.8]}, \frac{h_{3}}{[0.9,0.9]}, \frac{h_{4}}{[0.1,0.9]}\right\},\left\{\frac{c_{1}}{[0.7,0.8]} \frac{c_{2}}{[0.6,0.7]} \frac{c_{3}}{[0.3,0.5]}\right\}, \\
\left.\left\{\frac{v_{1}}{[0.3,0.6]} \frac{v_{2}}{[0,0]} \frac{v_{3}}{[1,1]}\right\}\right), d_{5},\left\{\frac{h_{1}}{[0.4,0.4]}, \frac{h_{2}}{[0.1,0.9]}, \frac{h_{3}}{[0,1]}, \frac{h_{4}}{[0.1,1]}\right\}, \\
\left.\left\{\frac{c_{1}}{[0.6,0.7]} \frac{c_{2}}{[0.8,0.8]} \frac{c_{3}}{[0.2,0.6]}\right\},\left\{\frac{v_{1}}{[0.2,0.9]} \frac{v_{2}}{[0.5,1]} \frac{v_{3}}{[0.5,0.5]}\right\}\right), \\
\left.d_{6},\left\{\frac{h_{1}}{[0.4,0.5]}, \frac{h_{2}}{[0.8,0.9]}, \frac{h_{3}}{[0.1,0.1]}, \frac{h_{4}}{[0.3,1]}\right\},\left\{\frac{c_{1}}{[0,1]} \frac{c_{2}}{[0,1]} \frac{c_{3}}{[0,1]}\right\},\left\{\frac{v_{1}}{[0,1]} \frac{v_{2}}{[0,1]} \frac{v_{3}}{[0,1]}\right\}\right) .
\end{gathered}
$$

Apply RMA to the second vague soft multiset part in $(H, D)_{1}$ to take the decision from the availability set $U_{2}$ and subtract the truth membership from the false-membership functions. The second resultant vague soft multiset part will be as in Table 4 .

Table 4: Representation of $U_{2}$-vague soft multiset part of $(H, D)_{1}$

\begin{tabular}{ccccccc}
\hline$U_{2}$ & $d_{1,1}$ & $d_{1,2}$ & $d_{1,3}$ & $d_{1,4}$ & $d_{1,5}$ & $d_{1,6}$ \\
\hline$c_{1}$ & 0 & -0.1 & 0 & -0.1 & -0.1 & 0 \\
$c_{2}$ & 0 & 0 & 0 & -0.1 & 0 & 0 \\
$c_{3}$ & 0 & -0.1 & 0 & -0.2 & -0.4 & 0 \\
\hline
\end{tabular}

The corresponding comparison table will be as in Table 5 . The row-sum, column-sum, and the score for each $c_{i}$ are in Table 6 .

From Table 6, the maximum score is 3 scored by $c_{2}$.

Redefine the vague soft multiset $(H, D)$ by keeping all values in each row where $c_{1}$ is maximum and replacing the values in the other rows by zero. 
Table 5: Comparison table of $U_{2}$-vague soft multiset part of $(H, D)_{1}$

\begin{tabular}{cccc}
\hline$U_{2}$ & $c_{1}$ & $c_{2}$ & $c_{3}$ \\
\hline$c_{1}$ & 6 & 3 & 6 \\
$c_{2}$ & 6 & 6 & 6 \\
$c_{3}$ & 4 & 3 & 6 \\
\hline
\end{tabular}

Table 6: Score of $U_{1}$-vague soft multiset part of $(H, D)$

\begin{tabular}{cccc}
\hline$U_{2}$ & Row sum $\left(r_{i}\right)$ & Column sum $\left(t_{i}\right)$ & Membership score $\left(s_{i}\right)$ \\
\hline$c_{1}$ & 15 & 14 & 1 \\
$c_{2}$ & 18 & 15 & 3 \\
$c_{3}$ & 13 & 18 & -5 \\
\hline
\end{tabular}

$(H, D)_{2}=\left\{d_{1},\left\{\frac{h_{1}}{[0.5,0.5]}, \frac{h_{2}}{[0.6,0.7]}, \frac{h_{3}}{[0.2,0.2]}, \frac{h_{4}}{[0,1]}\right\},\left\{\frac{c_{1}}{[0,1]} \frac{c_{2}}{[0,1]} \frac{c_{3}}{[0,1]}\right\}\right.$ $\left.\left\{\frac{v_{1}}{[0,1]} \frac{v_{2}}{[0,1]} \frac{v_{3}}{[0,1]}\right\}\right), d_{2},\left\{\frac{h_{1}}{[0.4,0.7]}, \frac{h_{2}}{[0.8,0.9]}, \frac{h_{3}}{[0.9,0.9]}, \frac{h_{4}}{[0.3,1]}\right\},\left\{\frac{c_{1}}{[0,1]} \frac{c_{2}}{[0,1]} \frac{c_{3}}{[0,1]}\right\}$, $\left.\left\{\frac{v_{1}}{[0,1]} \frac{v_{2}}{[0,1]} \frac{v_{3}}{[0,1]}\right\}\right), d_{3},\left\{\frac{h_{1}}{[0.2,0.7]}, \frac{h_{2}}{[0.1,0.1]}, \frac{h_{3}}{[0,0]}, \frac{h_{4}}{[0.9,0.9]}\right\},\left\{\frac{c_{1}}{[0,1]} \frac{c_{2}}{[0,1]} \frac{c_{3}}{[0,1]}\right\}$, $\left.\left\{\frac{v_{1}}{[0,1]} \frac{v_{2}}{[0,1]} \frac{v_{3}}{[0,1]}\right\}\right), d_{4},\left\{\frac{h_{1}}{[0.3,0.6]}, \frac{h_{2}}{[0.7,0.8]}, \frac{h_{3}}{[0.9,0.9]}, \frac{h_{4}}{[0.1,0.9]}\right\},\left\{\frac{c_{1}}{[0,1]} \frac{c_{2}}{[0,1]} \frac{c_{3}}{[0,1]}\right\}$, $\left.\left\{\frac{v_{1}}{[0,1]} \frac{v_{2}}{[0,1]} \frac{v_{3}}{[0,1]}\right\}\right), d_{5},\left\{\frac{h_{1}}{[0.4,0.4]}, \frac{h_{2}}{[0.1,0.9]}, \frac{h_{3}}{[0,1]}, \frac{h_{4}}{[0.1,1]}\right\}$, $\left.\left\{\frac{c_{1}}{[0.6,0.7]} \frac{c_{2}}{[0.8,0.8]} \frac{c_{3}}{[0.2,0.6]}\right\},\left\{\frac{v_{1}}{[0.2,0.9]} \frac{v_{2}}{[0.5,1]} \frac{v_{3}}{[0.5,0.5]}\right\}\right)$, $\left.d_{6},\left\{\frac{h_{1}}{[0.4,0.5]}, \frac{h_{2}}{[0.8,0.9]}, \frac{h_{3}}{[0.1,0.1]}, \frac{h_{4}}{[0.3,1]}\right\},\left\{\frac{c_{1}}{[0,1]} \frac{c_{2}}{[0,1]} \frac{c_{3}}{[0,1]}\right\},\left\{\frac{v_{1}}{[0,1]} \frac{v_{2}}{[0,1]} \frac{v_{3}}{[0,1]}\right\}\right)$.

Apply RMA to the third vague soft multiset part in $(H, D)_{2}$ to take the decision from the availability set $U_{3}$ and subtract the truth membership function from the false-membership functions. The third resultant vague soft multiset part will be as in Table 7 .

The corresponding comparison table will be as in Table 8 .

Compute the row-sum, column-sum, and the score for each $v_{i}$ as in Table 9.

From Table 9, the maximum score is 3 scored by $c_{2}$. Thus the most suitable 
Table 7: Representation of $U_{3}$-vague soft multiset part of $(H, D)_{2}$

\begin{tabular}{ccccccc}
\hline$U_{3}$ & $d_{1,1}$ & $d_{1,2}$ & $d_{1,3}$ & $d_{1,4}$ & $d_{1,5}$ & $d_{1,6}$ \\
\hline$v_{1}$ & 0 & 0 & 0 & 0 & -0.1 & 0 \\
$v_{2}$ & 0 & 0 & 0 & 0 & 0 & 0 \\
$v_{3}$ & 0 & 0 & 0 & 0 & -0.4 & 0 \\
\hline
\end{tabular}

Table 8: Comparison table of $U_{3}$-vague soft multiset part of $(H, D)_{2}$

\begin{tabular}{cccc}
\hline$U_{3}$ & $c_{1}$ & $c_{2}$ & $c_{3}$ \\
\hline$v_{1}$ & 6 & 5 & 6 \\
$v_{2}$ & 6 & 6 & 6 \\
$v_{3}$ & 5 & 5 & 6 \\
\hline
\end{tabular}

Table 9: Score table: $U_{1}$-vague soft multiset part of $(H, D)$

\begin{tabular}{cccc}
\hline$U_{2}$ & Row sum $\left(r_{i}\right)$ & Column sum $\left(t_{i}\right)$ & Membership score $\left(s_{i}\right)$ \\
\hline$v_{1}$ & 17 & 17 & 0 \\
$v_{2}$ & 18 & 16 & 2 \\
$v_{3}$ & 16 & 18 & -2 \\
\hline
\end{tabular}

decision for the person is $\left(h_{3}, c_{2}, v_{2}\right)$. This means that the person will chose venue Shah Alam $h_{3}$, to buy DC machine $c_{2}$ and heavy trucks $v_{2}$.

\section{Conclusion}

The concept of vague soft multiset theory is proposed and an application to solve a decision making problem is illustrated. It is also desirable to further explore the applications of using the vague soft multiset approach to solve real world decision making problems. A potential area of research involves extending our work to study the relationship between soft set, multiset and vague soft set. 


\section{Acknowledgments}

The authors would like to acknowledge the financial support received from Universiti Kebangsaan Malaysia under the research grant BKBP-FST-K005560.

\section{References}

[1] N. Hassan, K.B. Hassan, S.S Yatim and S.A. Yusof, Optimizing fertilizer compounds and minimizing the cost of cucumber production using the goal programming approach, American-Eurasian Journal of Sustainable Agriculture, 7 (2) (2013), 45-49.

[2] N. Hassan, H.H.M. Hamzah and S.M.M. Zain, A goal programming approach for rubber production in Malaysia, American-Eurasian Journal of Sustainable Agriculture, 7 (2) (2013), 50-53.

[3] N. Hassan, N. Ahmad and W.M.W. Aminuddin, Selection of mobile network operator using analytic hierarchy process, Advances in Natural and Applied Sciences, 7 (1) (2013), 1-5.

[4] N. Hassan, A.H.M. Pazil, N.S. Idris and N.F. Razman, A goal programming model for bakery production, Advances in Environmental Biology, 7 (1) (2013), 187-190.

[5] N. Hassan, S. Safiai, N.H.M. Raduan and Z. Ayop, Goal programming formulation in nutrient management for chilli plantation in Sungai Buloh, Malaysia, Advances in Environmental Biology, 6 (12) (2012), 4008-4012.

[6] N. Hassan and B.A. Halim, Mathematical modelling approach to the management of recreational tourism activities at Wetland Putrajaya (in Malay), Sains Malaysiana, 41 (9) (2012), 1155-1161.

[7] N. Hassan and L.L. Loon, Goal programming with utility function for funding allocation of a university library, Applied Mathematical Sciences, 6 (110) (2012), 5487-5493.

[8] N. Hassan, L.W. Siew and S.Y. Shen, Portfolio decision analysis with maximin criterion in the Malaysian stock market, Applied Mathematical Sciences, 6 (110) (2012), 5483- 5486. 
[9] N. Hassan and S. Sahrin, A mathematical model of nutrient management for pineapple cultivation in Malaysia, Advances in Environmental Biology, 6 (5) (2012), 1868-1872.

[10] N. Hassan and Z. Ayop, A goal programming approach for food product distribution of small and medium enterprises, Advances in Environmental Biology, 6 (2) (2012), 510-513.

[11] N. Hassan and S.B.M. Basir, A goal programming model for scheduling political campaign: A case study in Kabupaten Kampar, Riau, Indonesia (in Malay), Journal of Quality Measurement and Analysis, 5 (2) (2009), 99-107.

[12] N. Hassan, D.F. Azmi, T.S. Guan and L.W. Hoe, A goal programming approach for library acquisition allocation, Applied Mathematical Sciences, 7 (140) (2013), 6977-6981.

[13] N. Hassan and M.M. Tabar, The relationship of multiple objectives linear programming and data envelopment analysis, Australian Journal of Basic and Applied Sciences, 5 (11) (2011), 1711-1714.

[14] N. Hassan, M.M. Tabar and P. Shabanzade, Resolving multi objectives resource allocation problem based on inputs and outputs using data envelopment analysis method, Australian Journal of Basic and Applied Sciences, 4 (10) (2010), 5320-5325.

[15] N. Hassan, M.M. Tabar and P. Shabanzade, A ranking model of data envelopment analysis as a centralized multi objective resource allocation problem tool, Australian Journal of Basic and Applied Science, 4 (10) (2010), 5306-5313.

[16] L.A. Zadeh, Fuzzy sets, Information and Control, 8 (3) (1965), 338-353.

[17] S. Alkhazaleh. A. R. Salleh and N. Hassan, Soft multisets theory, Applied Mathematical Sciences, 5 (72) (2011), 3561-3573.

[18] S. Alkhazaleh. A. R. Salleh and N. Hassan, Possibility fuzzy soft set, Advances in Decision Sciences, Article ID 479756, 18 pages (2012), doi:10.1155/2011/479756.

[19] S. Alkhazaleh. A. R. Salleh and N. Hassan, Fuzzy parameterized intervalvalued fuzzy soft set, Applied Mathematical Sciences, 5 (67) (2011), 33353346. 
[20] A.R. Salleh, S. Alkhazaleh, N. Hassan and A.G. Ahmad, Multiparameterized soft set, Journal of Mathematics and Statistics, 8 (1) (2012), 92-97.

[21] K. Alhazaymeh, S.A. Halim, A. R. Salleh, and N. Hassan, Soft intuitionistic fuzzy sets, Applied Mathematical Sciences, 6 (54) (2012), 2669-2680.

[22] K. Alhazaymeh and N. Hassan, Generalized vague soft set and its applications, International Journal of Pure and Applied Mathematics, 77 (3) (2012), 391-401.

[23] K. Alhazaymeh and N. Hassan, Possibility vague soft set and its application in decision making, International Journal of Pure and Applied Mathematics, 77 (4) (2012), 549-563.

[24] K. Alhazaymeh and N. Hassan, Interval-valued vague soft sets and its application, Advances in Fuzzy Systems, Article ID 208489, 7 pages (2012), doi:10.1155/2012/208489.

[25] N. Hassan and K. Alhazaymeh, Vague soft expert set theory, AIP Conference Proceedings, 1522 (2013), 953-958 ; doi: 10.1063/1.4801233.

[26] K.Alhazaymeh and N.Hassan, Possibility interval-valued vague soft set, Applied Mathematical Sciences, 7 (140) (2013), 6989-6994.

[27] K.Alhazaymeh and N.Hassan, Generalized interval-valued vague soft set, Applied Mathematical Sciences, 7 (140) (2013), 6983-6988.

[28] K.Alhazaymeh and N.Hassan, Generalized vague soft expert set, International Journal of Pure and Applied Mathematics, (in press).

[29] K.Alhazaymeh and N.Hassan, Application of generalized vague soft expert set in decision making, International Journal of Pure and Applied Mathematics, (in press).

[30] K.Alhazaymeh and N.Hassan, Mapping on generalized vague soft expert set, International Journal of Pure and Applied Mathematics, (in press).

[31] M. Varnamkhasti and N. Hassan, A hybrid of adaptive neuro-fuzzy inference system and genetic algorithm, Journal of Intelligent and Fuzzy Systems, 25 (3) (2013), 793-796.

[32] M. Varnamkhasti and N. Hassan, Neurogenetic algorithm for solving combinatorial engineering problems, Journal of Applied Mathematics, Article ID 253714, 12 pages (2012), doi:10.1155/2012/253714 
[33] F. Adam and N. Hassan, Multi Q-fuzzy parameterized soft set and its application, Journal of Intelligent and Fuzzy System, (in press).

[34] D. Molodtsov, Soft set theory-first result, Computers and Mathematics with Applications, 37 (1999), 19-31. 
